\title{
QualiSex: estudo da associação entre a qualidade de vida e a sexualidade nos idosos numa população do Porto
}

Mariana Cambão, Lígia Sousa, Miguel Santos, Sandra Mimoso, Sara Correia, Dilermando Sobral

\section{RESUMO}

Objetivos: Caracterizar a sexualidade na população idosa de uma área urbana de Portugal e relacioná-la com a qualidade de vida. Tipo de estudo: Estudo observacional, transversal e descritivo, com componente analítica.

Local: Unidade de Saúde Familiar de Ramalde (USFR), Agrupamentos de Centros de Saúde Porto Ocidental.

População: Utentes inscritos na USFR, com idade $\geq 65$ anos.

Métodos: Obteve-se uma amostragem aleatória através da ferramenta eletrónica disponível em www.random.org. O tamanho amostral foi calculado com recurso ao programa OpenEpi ${ }^{\circledR}$. Os utentes foram convidados telefonicamente a participar no estudo. Para aqueles que aceitaram agendou-se a realização de entrevista presencial, com um questionário estruturado em duas partes: um questionário elaborado pelos investigadores (variáveis sociodemográficas e variáveis relacionadas com a sexualidade); outra que corresponde ao questionário SF-12. A análise dos dados foi realizada através de estatística descritiva e inferencial, recorrendo ao programa SPSS ${ }^{\circledR}$, v. 24.

Resultados: A amostra incluiu 213 utentes, maioritariamente mulheres (59,2\%), com idade média de 73 anos (desvio-padrão=5,96). Metade dos utentes tinha vida sexual ativa (maioritariamente homens) e $75 \%$ referiu satisfação com a vida sexual. O género masculino atribuiu maior importância à vida sexual. Verificou-se uma associação entre uma vida sexual ativa e algumas dimensões do SF-12, em ambos os géneros. A satisfação das mulheres com a sua vida sexual relacionou-se com a dimensão «saúde mental».

Conclusões: Este estudo pioneiro focou um tema que tem sido negligenciado. Demonstrou-se a importância da sexualidade numa faixa etária por vezes considerada «assexuada» e o seu impacto na qualidade de vida, o que reforça a pertinência da abordagem destas questões nas consultas de medicina geral e familiar.

Palavras-chave: Idoso; Qualidade de vida; Sexualidade.

\section{INTRODUÇÃO}

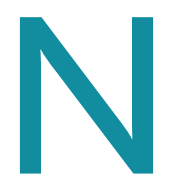

o século XX assistiu-se, de uma forma geral, a uma melhoria dos cuidados de saúde, em particular nos países desenvolvidos e Portugal não foi exceção. O desenvolvimento e a melhoria da educação e das ciências médicas foram muito importantes no aumento da esperança média de vida. Concomitantemente, devido à diminuição da taxa de natalidade, assistiu-se a uma inversão da pirâmide populacional com os indivíduos a viverem mais tempo e a procurarem viver com melhor qualidade.
Diversos autores têm procurado estudar vários parâmetros que definem qualidade de vida, havendo alguns questionários que permitem, de uma forma validada, quantificar a qualidade de vida individual. ${ }^{1-2} \mathrm{Um}$ deles é o SF-12, que mede os oito conceitos normalmente presentes em pesquisas e amplamente utilizados: «Função Física», «Desempenho Físico», «Dor», «Saúde em Geral», «Vitalidade», «Função Social», «Desempenho Emocional»e «Saúde Mental». Foi validado para a população portuguesa por Pedro Lopes Ferreira e deriva do instrumento SF-36. ${ }^{3-4}$ Porém, poucos estudos se têm debruçado sobre a sexualidade e a forma como ela é vivida, particularmente nas pessoas mais 
idosas, tendo em conta o seu reflexo na qualidade de vida. Numa revisão de 2012 foi notado que uma vida sexual regular está associada a um bom estado de saúde mental e físico. ${ }^{5}$ Na literatura, a sexualidade tem sido principalmente abordada sob o aspeto fisiológico, relatando as perdas que ocorrem com o processo de envelhecimento; outras variáveis, como a aceitação da aparência física, o sedentarismo, o estado civil e o relacionamento social, que também influenciam a satisfação das pessoas idosas no que toca à sua sexualidade, pouco têm sido avaliadas. ${ }^{6}$

O organismo sofre alterações fisiológicas com o avançar da idade e a forma como é vivida a sexualidade também evolui de maneira distinta nos dois sexos. ${ }^{7-8}$ A maioria dos indivíduos idosos encontra-se envolvida em relações matrimoniais ou em outro tipo de relações íntimas e considera que a sexualidade é uma parte importante da vida. ${ }^{9}$ Apesar da prevalência da atividade sexual decrescer com a idade, uma parte considerável da população idosa pratica sexo vaginal, oral e/ou masturbação, mesmo na oitava e nona década de vida. ${ }^{9}$ Esta noção de que a sexualidade no idoso é tida como importante para a sua vida revela-se no facto de que a frequência de a atividade sexual não decrescer substancialmente até em idades avançadas (74 anos) quando comparada com populações mais jovens, apesar de uma grande prevalência $(>50 \%)$ de problemas relacionados com a sexualidade. ${ }^{5,9} \mathrm{~A}$ partir da faixa etária entre os 75 e os 85 anos é que se começa a notar um decréscimo na frequência da atividade sexual; contudo, mesmo neste grupo etário, $54 \%$ dos indivíduos sexualmente ativos relatam uma frequência de relações sexuais de duas a três vezes por mês. ${ }^{9}$ Um outro estudo mostrou que existe uma presença significativa de atividade, desejo e função sexual acima dos 50 anos de idade, observando-se um decréscimo acima dos 60 anos, maioritariamente nas mulheres. ${ }^{10}$ Mais de metade das mulheres com mais de 60 anos mantém vida sexual ativa se coabitarem com um companheiro e os fatores psicossociais (qualidade do relacionamento e da comunicação entre o casal, etc.) têm mais impacto na satisfação sexual do que propriamente o envelhecimento. ${ }^{11}$

Um estudo levado a cabo em homens e mulheres com mais de 50 anos (idade média de 67 anos) demonstrou que apenas numa minoria se verificava um impacto negativo do estado de saúde na atividade se- xual e, quando isso acontecia, os fatores que demonstraram associação foram a idade avançada, o género masculino, o peso e uma baixa pontuação no SF-36 nas dimensões física e mental. ${ }^{12}$ Existem muitos estudos que avaliam o impacto negativo de várias doenças e de medicação na função sexual em pessoas acima dos 50 anos; no entanto, existe pouca investigação acerca da forma como a sexualidade é vivida em idades mais avançadas, revelando uma lacuna no conhecimento por parte da comunidade científica. ${ }^{5}$

São objetivos do presente estudo: caracterizar a sexualidade de uma amostra de uma população idosa do Porto e relacioná-la com a sua qualidade de vida.

\section{MÉTODOS}

Foi realizado um estudo transversal (observacional). Procedeu-se a uma aleatorização de todos os utentes com idade igual ou superior a 65 anos inscritos na Unidade de Saúde Familiar de Ramalde (USFR) em abril de 2014, através da ferramenta eletrónica disponível em www.random.org. Definiram-se, como critérios de inclusão, ser utente da USFR e ter idade igual ou superior a 65 anos. Foram critérios de exclusão: existência de défices comunicacionais (nomeadamente surdez, mudez ou outros défices de compreensão evidentes); impossibilidade de contacto durante o período de recolha de dados; dependência de terceiros para as atividades básicas da vida diária.

O tamanho amostral estipulado (340 utentes) foi calculado recorrendo ao programa OpenEpi®, considerando a população de utentes com idade igual ou superior a 65 anos inscritos na USFR em abril de 2014 ( $n=2.891$ ), uma prevalência estimada de $50 \%$, uma margem de erro de $5 \%$ e um nível de confiança de $95 \%$.

Foram verificadas as datas das próximas consultas para todos os indivíduos da amostra, separando-a em dois grupos - o grupo dos indivíduos com consulta programada dentro dos três meses posteriores à amostragem e o grupo que não tinha consulta nesse período de tempo. Todos os indivíduos foram contactados telefonicamente de forma a serem informados acerca da realização do estudo e a averiguar do seu interesse em participar. O contacto telefónico obedeceu a uma estruturação predefinida. Efetuaram-se até três tentativas de contacto telefónico em três ocasiões distintas. Os indivíduos com consulta programada foram submetidos 
ao questionário, após consentimento informado, na data da mesma.

Nos indivíduos do segundo grupo averiguou-se a possibilidade de deslocação à USFR para a aplicação do questionário. Na impossibilidade dessa deslocação foi oferecida a alternativa de dois dos investigadores se deslocarem a casa do indivíduo e aplicar o questionário no domicílio, sendo marcado um dia e uma hora para essa deslocação. A entrevista foi preferencialmente realizada por um investigador do mesmo sexo que o entrevistado.

O questionário foi aplicado entre junho e dezembro de 2014.

As variáveis em estudo foram obtidas através de um questionário com duas partes: uma, elaborada pelos autores, que aborda variáveis sociodemográficas e variáveis relacionadas com a sexualidade; outra constituída pelo questionário SF-12, que avalia a qualidade de vida.

As variáveis sociodemográficas exploradas foram: género, idade, escolaridade (analfabeto; ensino primário; ensino básico; ensino secundário; ensino superior), situação conjugal [solteiro(a), união de facto/casado(a), divorciado(a), viúvo(a)], rendimento mensal do agregado familiar (até 500 euros; entre 500-1.500 euros; mais de 1.500 euros) e constituição do agregado familiar (unitário; companheiro ou cônjuge; outros familiares; outros não familiares; companheiro ou cônjuge e outros familiares).

A sexualidade dos idosos foi avaliada através de seis questões que pretendiam aprofundar a importância atribuída à vida sexual (não importante, pouco importante, importante, muito importante), a existência de uma vida sexual ativa (sim, não), a frequência da vida sexual atual (pelo menos uma vez por ano; pelo menos uma vez a cada seis meses; pelo menos uma vez por mês; pelo menos uma vez por semana; mais do que uma vez por semana), os parceiros sexuais [namorado(a), esposo(a), ocasional, profissional, masturbação, outro], a satisfação com a vida sexual [satisfeito(a), insatisfeito(a)] e a preferência sexual (mesmo género, género oposto). Os autores consideraram «vida sexualmente ativa» a existência de qualquer tipo de prática destinada à partilha ou fruição de prazer sexual (masturbação, relações sexuais com ou sem penetração).

O SF-12 contempla a avaliação de oito dimensões:
«Função Física», «Desempenho Físico», «Dor», «Saúde em Geral», «Vitalidade», «Funcionamento Social», «Desempenho Emocional» e «Saúde Mental».

A «Função Física» pretende avaliar o impacto na qualidade de vida das limitações físicas, em situações do quotidiano, como tratar da higiene pessoal, vestir-se sozinho(a), transportar os sacos das compras, ajoelhar-se ou caminhar uma certa distância. $\mathrm{O}$ «Desempenho Físico» avalia o impacto das limitações em saúde devidas a problemas físicos. A dimensão «Dor» avalia o desconforto causado pela dor e de que forma é que esta interfere com o trabalho habitual. A dimensão «Saúde em Geral» mede a perceção holística da saúde, considerando a saúde atual e a resistência à doença. A «Vitalidade» avalia a perceção dos níveis de energia e de fadiga. $\mathrm{O}$ «Funcionamento Social» explora a quantidade e a qualidade das atividades sociais e o impacto dos problemas físicos e emocionais nas mesmas. $\mathrm{O}$ «Desempenho Emocional» avalia o impacto das limitações em saúde devido a problemas emocionais. Por último, a «Saúde Mental» contempla os conceitos de ansiedade, depressão, perda de controlo comportamental ou emocional e de bem-estar psicológico. ${ }^{13}$

Estas oito dimensões podem ser agregadas em duas medidas de sumário física e mental, designadas por «Medida de Sumário Física»e «Medida de Sumário Mental». A Medida de Sumário Física é constituída pelas dimensões «Função Física», «Desempenho Físico», «Dor» e «Saúde em Geral». A Medida de Sumário Mental contempla as dimensões «Saúde Mental», «Desempenho Emocional», «Função Social» e «Vitalidade». ${ }^{13}$

Para cada dimensão do SF-12 a pontuação oscila entre 0 e 100, com os extremos a corresponderem, respetivamente, à pior e à melhor qualidade de vida relacionada com a saúde. ${ }^{13}$

O preenchimento foi efetuado por entrevista para obviar as dificuldades de leitura e de compreensão associadas à população em estudo.

Para a análise estatística dos dados foi usado o SPSS ${ }^{\circledR}$ v. 24 (teste $t$, ANOVA, Teste Qui-quadrado e Teste de Fisher).

Obteve-se parecer favorável da Comissão de Ética da ARS Norte e da Comissão Nacional de Proteção de Dados.

Foram preservados o anonimato e a confidencialidade dos dados recolhidos através da atribuição de um 
código alfanumérico a cada questionário, sem correspondência com o nome e morada do participante, e a compilação dos dados obtidos foi salvaguardada em computador protegido com palavra-passe.

\section{RESULTADOS}

Foram incluídos 213 participantes, de um total de 2.891 utentes com idade igual ou superior a 65 anos, inscritos na USFR à data do estudo. Verificou-se o predomínio do género feminino (59\% de mulheres) em proporções semelhantes à da população idosa (62\% de mulheres). Os participantes tinham uma média de idades de 73,4 anos [desvio-padrão (DP) $=6,0$ ], próxima da média de idades da população $(75,9$ anos; $\mathrm{DP}=7,5)$. No Quadro I encontra-se representada a distribuição da amostra por género e grupo etário: o género feminino é o mais prevalente, exceto nas faixas etárias dos 75-79 anos e $\geq 95$ anos. No Quadro II ilustram-se os dados relativos à população: há um predomínio do género feminino em todas as faixas etárias.

Os não-respondentes foram 217; este número inclui os utentes que recusaram participar e os que foram excluídos de acordo com os critérios estabelecidos. O principal motivo de exclusão foi a impossibilidade de contacto telefónico. No total, os autores contactaram ou tentaram contactar (nas situações em que houve impossibilidade de contacto telefónico em três ocasiões distintas) 430 utentes, mas destes apenas 213 foram incluídos no estudo. Relativamente à caracterização dos não-respondentes, tinham uma idade média de 77,9 anos $(\mathrm{DP}=7,4)$ e $63 \%$ eram do género feminino. $\mathrm{O}$ grupo etário predominante foi dos 70-79 anos Quadro III.

As variáveis sociodemográficas e familiares estudadas estão descritas no Quadro IV.

Relativamente à escolaridade, a maioria das mulheres apresentava o ensino primário (51,6\%). No género masculino, apesar de ser igualmente mais comum o ensino primário (36,6\%), verificou-se uma maior prevalência dos níveis de ensino

\begin{tabular}{|c|c|c|c|}
\hline \multicolumn{4}{|c|}{$\begin{array}{l}\text { QUADRO II. Caracterização da popul } \\
\text { etário (anos) }\end{array}$} \\
\hline \multirow{2}{*}{$\begin{array}{l}\text { Grupo etário } \\
\text { (anos) }\end{array}$} & \multicolumn{2}{|c|}{ Género } & \multirow[b]{2}{*}{ Total (n/\%) } \\
\hline & Feminino (n/\%) & Masculino (n/\%) & \\
\hline $65-69$ & $414 / 23,1$ & $317 / 28,8$ & $731 / 25,3$ \\
\hline $70-74$ & $361 / 20,1$ & $250 / 22,7$ & $611 / 21,1$ \\
\hline $75-79$ & $361 / 20,1$ & $229 / 20,8$ & $590 / 20,4$ \\
\hline $80-84$ & $350 / 19,5$ & $177 / 16,1$ & $527 / 18,2$ \\
\hline $85-89$ & $227 / 12,7$ & $92 / 8,4$ & $319 / 11,0$ \\
\hline $90-94$ & $61 / 3,4$ & $33 / 3,0$ & $94 / 3,3$ \\
\hline$\geq 95$ & $18 / 1,0$ & $1 / 0,09$ & $19 / 0,66$ \\
\hline
\end{tabular}

QUADRO III. Caracterização dos não respondentes por género e grupo etário (anos)

\begin{tabular}{l|c|c|c|}
\multirow{2}{*}{$\begin{array}{l}\text { Grupo etário } \\
\text { (anos) }\end{array}$} & \multicolumn{2}{|c|}{ Género } & \multirow{2}{*}{} \\
\cline { 2 - 3 } & $\begin{array}{c}\text { Feminino (n/\%) } \\
137\end{array}$ & $\begin{array}{c}\text { Masculino (n/\%) } \\
\text { Total (n/\%) } \\
\mathbf{2 1 7}\end{array}$ \\
\hline $65-69$ & $12 / 8,7$ & $20 / 25$ & $32 / 14,7$ \\
\hline $70-74$ & $36 / 26,3$ & $15 / 18,8$ & $51 / 23,5$ \\
\hline $75-79$ & $29 / 21,2$ & $19 / 23,8$ & $48 / 22,1$ \\
\hline $80-84$ & $24 / 17,5$ & $14 / 17,5$ & $38 / 17,5$ \\
\hline $85-89$ & $26 / 19,0$ & $7 / 8,7$ & $33 / 15,2$ \\
\hline $90-94$ & $7 / 5,1$ & $3 / 3,7$ & $10 / 4,6$ \\
\hline$\geq 95$ & $3 / 2,2$ & $2 / 2,5$ & $5 / 2,3$ \\
\hline
\end{tabular}




\begin{tabular}{|c|c|c|c|}
\hline \multicolumn{4}{|c|}{$\begin{array}{l}\text { QUADRO IV. Caracterização da amostra: variáveis } \\
\text { sociodemográficas e familiares }\end{array}$} \\
\hline \multirow[b]{2}{*}{ Variável } & \multicolumn{2}{|c|}{ Género } & \multirow[b]{2}{*}{$\begin{array}{l}\text { Total } \\
(\%)\end{array}$} \\
\hline & $\begin{array}{l}\text { Feminino } \\
(\%)\end{array}$ & $\begin{array}{l}\text { Masculino } \\
\text { (\%) }\end{array}$ & \\
\hline Escolaridade & $n=126$ & $n=87$ & \\
\hline Analfabeto & 1,6 & 1,1 & 1,4 \\
\hline Ensino primário & 51,6 & 35,6 & 45 \\
\hline Ensino básico & 19,0 & 15,0 & 17,4 \\
\hline Ensino secundário & 13,5 & 26,4 & 18,8 \\
\hline Ensino superior & 14,3 & 21,9 & 17,4 \\
\hline Situação conjugal & $n=126$ & $n=87$ & \\
\hline Solteiro(a) & 11,1 & 1,1 & 7,0 \\
\hline União de facto/casado(a) & 55,6 & 92,0 & 70,4 \\
\hline Divorciado(a) & 6,3 & 2,3 & 4,8 \\
\hline Viúvo(a) & 27,0 & 4,6 & 17,8 \\
\hline Rendimento mensal do agregado & $n=125^{*}$ & $n=87$ & \\
\hline Até $€ 500,00$ & 32 & 11,5 & 23,6 \\
\hline$€ 500,00 \mathrm{a} € 1.500,00$ & 49,6 & 54 & 51,4 \\
\hline Mais de $€ 1.500,00$ & 18,4 & 34,5 & 25 \\
\hline Agregado familiar & $n=126$ & $n=87$ & \\
\hline Sozinho & 27,0 & 6,9 & 18,8 \\
\hline Companheiro ou cônjuge & 54,8 & 86,2 & 56,3 \\
\hline Outros familiares & 26,2 & 19,5 & 12,2 \\
\hline Outros não familiares & 1,6 & 1,1 & 1,4 \\
\hline $\begin{array}{l}\text { Companheiro ou cônjuge }+ \\
\text { outros familiares }\end{array}$ & 9,5 & 13,8 & 11,3 \\
\hline
\end{tabular}

Nota: *Uma das participantes não respondeu a esta questão do questionário.
Quanto à constituição do agregado familiar, a maioria dos homens (86\%) e mulheres (55\%) coabitava com o respetivo companheiro ou cônjuge. Entre os participantes que viviam sozinhos ou com outros familiares encontram-se mais mulheres.

Os resultados obtidos relativamente à sexualidade dos idosos encontram-se descritos no Quadro V.

Os homens atribuíram maior importância à vida sexual ( $p<0,001$; Teste Qui-quadrado) e referiram ter vida sexual ativa em maior proporção do que as mulheres ( $p<0,001$; Teste Qui-quadrado). A média da importância atribuída pelas mulheres à vida sexual $(2,42$; IC95\% [2,24-2,61]) foi significativamente menor do que a atribuída pelos homens (3,11; IC95\% [2,95-3,28]).

Os idosos que referiram ter pelo menos um tipo de parceiro sexual [namorado(a), esposo(a), ocasional e/ou profissional] ou que recorriam à masturbação atribuíram maior importância à vida sexual (diferença de médias $=0,559$; IC95\% [0,290-0,827]; $p<0,001$; teste $t$ ). Não se encontraram diferenças com significado estatístico relativamente à satisfação com a vida sexual entre os grupos com diferentes tipos de parceiros sexuais (diferenças entre médias=0,07; IC95\% [-0,29$0,15])$.

A importância atribuída à vida sexual decresce com o avançar da idade ( $p<0,001$; ANOVA) e os idosos com vida sexual ativa têm uma média de idades (72 anos) inferior aos que não têm vida sexual ativa (75 anos), sendo esta diferença estatisticamente significativa ( $p<0,01$; teste $t$ ).

O estado civil dos idosos parece influenciar a importância atribuída à vida sexual e a presença ou não de uma vida sexual ativa. Os participantes que viviam casados/em união de facto conferiram uma maior importância à vida sexual relativamente aos idosos solteiros, de forma estatisticamente significativa $(p<0,05$; teste $t$ para amostras independentes). Apesar dos viúvos atribuírem uma menor importância à vida sexual, as diferenças encontradas não foram estatisticamente significativas. Há uma maior proporção de idosos casados/em união de facto com vida sexual ativa $(70 \%)$, relativamente aos solteiros $(8,3 \%)$, divorciados $(10 \%)$ 
ou viúvos $(16,1 \%)$, sendo estas diferenças estatisticamente significativas $(p<0,01$; teste Qui-quadrado).

No que respeita à frequência da atividade sexual, a maioria dos participantes tinha relações sexuais "pelo menos uma vez por semana" ou "pelo menos uma vez por mês". Os homens tinham uma maior frequência de atividade sexual ( $p=0,04$; Teste de Fisher) do que as mulheres. Os idosos que referiram apresentar uma maior frequência de atividade sexual também atribuíram uma maior importância à vida sexual ( $p<0,001$; Teste de Fisher).

A maioria dos idosos entrevistados tinha como parceiro sexual o respetivo cônjuge.

Relativamente à satisfação com a atividade sexual (esta variável apenas foi inquirida nos idosos com vida sexual ativa), grande parte dos idosos referiu encontrarse satisfeito. Não se identificaram diferenças estatisticamente significativas entre géneros a este respeito ( $p>0,05$; Teste Qui-quadrado) nem se verificou a associação entre idade e satisfação com a vida sexual ( $p=0,463$; teste $t$ ). O estado civil dos participantes parece condicionar a satisfação com a vida sexual: os idosos solteiro(a)s e viúvo(a)s apresentavam maior satisfação do que os casados/em união de facto ou divorciados ( $p<0,05$; ANOVA).

Quando avaliada a associação entre escolaridade e importância atribuída à vida sexual, detetou-se que os idosos que possuíam maior escolaridade conferiam maior importância à vida sexual ( $p<0,01$; Teste Qui-quadrado). Constatou-se uma situação análoga relativamente ao rendimento mensal do agregado familiar: as remunerações mais elevadas associaram-se à atribuição de uma maior importância à vida sexual $(p<0,001$; Teste Qui-quadrado).

Relativamente à orientação sexual, todos os participantes afirmaram ser heterossexuais.

Verificou-se que, em ambos os géneros, os indivíduos com vida sexual ativa apresentavam uma pon-

\begin{tabular}{|c|c|c|c|}
\hline \multirow[b]{2}{*}{ Variável } & \multicolumn{2}{|c|}{ Género } & \multirow[b]{2}{*}{$\begin{array}{c}\text { Subtotal } \\
(\%)\end{array}$} \\
\hline & $\begin{array}{c}\text { Feminino } \\
(\%)\end{array}$ & $\begin{array}{c}\text { Masculino } \\
(\%)\end{array}$ & \\
\hline Importância atribuída à vida sexual & $n=126$ & $n=87$ & \\
\hline Não importante & 26,2 & 5,7 & 17,8 \\
\hline Pouco importante & 21,4 & 6,9 & 15,5 \\
\hline Importante & 36,5 & 57,5 & 45,1 \\
\hline Muito importante & 15,9 & 29,9 & 21,6 \\
\hline Vida sexual ativa & $n=126$ & $n=87$ & \\
\hline Sim & 36,5 & 70,1 & 50,2 \\
\hline Não & 63,5 & 29,9 & 49,8 \\
\hline Frequência da vida sexual & $n=46$ & $n=61$ & $n=107$ \\
\hline Pelo menos 1 vez por ano & 4,3 & 0 & 1,9 \\
\hline Pelo menos 1 vez a cada 6 meses & 19,6 & 11,5 & 15,0 \\
\hline Pelo menos 1 vez por mês & 32,6 & 37,7 & 35,5 \\
\hline Pelo menos 1 vez por semana & 43,5 & 39,3 & 41,1 \\
\hline Mais do que 1 vez por semana & 0 & 11,5 & 6,5 \\
\hline Parceiro sexual & $n=46$ & $n=61$ & $n=107$ \\
\hline Namorado(a) & 6,5 & 6,6 & 6,5 \\
\hline Esposo(a) & 91,3 & 86,9 & 88,8 \\
\hline Ocasional & 0 & 6,6 & 3,7 \\
\hline Profissional & 0 & 1,6 & 0,9 \\
\hline Masturbação & 6,5 & 21,3 & 15,0 \\
\hline Outro & 0 & 1,6 & 0,9 \\
\hline Satisfação com a vida sexual & $n=46$ & $n=61$ & $n=107$ \\
\hline Satisfeito & 78,3 & 72,1 & 74,8 \\
\hline Insatisfeito & 21,7 & 27,9 & 25,2 \\
\hline Preferência sexual & $n=126$ & $n=87$ & $n=113$ \\
\hline Género oposto & 100 & 100 & 100 \\
\hline
\end{tabular}

tuação superior nas diferentes dimensões do SF-12. Contudo, estas diferenças apenas atingiram o limiar da significância estatística nas dimensões «Função Física» ( $p<0,01$; teste $t)$, «Desempenho Físico» $(p<0,05$; teste $t)$, «Saúde em Geral» $(p<0,01$; teste $t)$ e «Funcionamento Social» $(p<0,01$; teste $t)$ no género feminino (Quadro VI); e nas dimensões «Função Física» $(p<0,01$; teste $t)$, «Desempenho Físico» $(p<0,05$; teste $t)$, «Saúde em Geral» $(p<0,05$; teste $t)$, «Vitalidade» $(p<0,01$; teste $t)$ $\mathrm{e}$ «Desempenho Emocional» $(p<0,05$; teste $t)$ no género masculino (Quadro VII). 


\begin{tabular}{|c|c|c|c|}
\hline \multicolumn{4}{|c|}{$\begin{array}{l}\text { QUADRO VI. Associação entre vida sexual ativa e as diferentes } \\
\text { dimensões do SF-12, no género feminino }\end{array}$} \\
\hline Dimensão do SF-12 & $\begin{array}{l}\text { Vida sexual } \\
\text { ativa }\end{array}$ & $\begin{array}{l}\text { Sem vida } \\
\text { sexual ativa }\end{array}$ & Valor de $p$ \\
\hline Função Física & 65,76 & 46,88 & $<0,01$ \\
\hline Desempenho Físico & 72,55 & 57,03 & $<0,05$ \\
\hline Dor & 63,04 & 53,75 & $>0,05$ \\
\hline Saúde em Geral & 40,98 & 24,81 & $<0,01$ \\
\hline Vitalidade & 55,43 & 47,5 & $>0,05$ \\
\hline Funcionamento Social & 77,72 & 56,96 & $<0,01$ \\
\hline Desempenho Emocional & 77,45 & 69,68 & $>0,05$ \\
\hline Saúde Mental & 63,59 & 57,03 & $>0,05$ \\
\hline Medida de Sumário Mental & 49,72 & 46,74 & $>0,05$ \\
\hline
\end{tabular}

\begin{tabular}{|c|c|c|c|}
\hline \multicolumn{4}{|c|}{$\begin{array}{l}\text { QUADRO VII. Associação entre vida sexual ativa e as diferentes } \\
\text { dimensões do SF-12, no género masculino }\end{array}$} \\
\hline Dimensão do SF-12 & $\begin{array}{c}\text { Vida sexual } \\
\text { ativa }\end{array}$ & $\begin{array}{c}\text { Sem vida } \\
\text { sexual ativa }\end{array}$ & Valor de $p$ \\
\hline Função Física & 81,97 & 57,69 & $<0,01$ \\
\hline Desempenho Físico & 81,56 & 67,79 & $<0,05$ \\
\hline Dor & 72,95 & 69,23 & $>0,05$ \\
\hline Saúde em Geral & 52,79 & 37,31 & $<0,05$ \\
\hline Vitalidade & 62,3 & 43,27 & $<0,01$ \\
\hline Funcionamento Social & 81,56 & 72,12 & $>0,05$ \\
\hline Desempenho Emocional & 83,4 & 71,63 & $<0,05$ \\
\hline Saúde Mental & 71,72 & 62,02 & $>0,05$ \\
\hline Medida de Sumário Mental & 50,88 & 47,87 & $>0,05$ \\
\hline
\end{tabular}

Quando avaliada a associação entre a satisfação com a vida sexual e as diferentes dimensões do SF-12 também se observou a tendência entre os «satisfeitos» para pontuações mais elevadas. No género feminino a diferença entre os grupos apenas foi estatisticamente significativa na dimensão «Saúde Mental» $(p<0,05$; teste $t)$ $\mathrm{e}$, no género masculino, não se identificaram diferenças estatisticamente significativas entre o grupo dos «satisfeitos»e dos «insatisfeitos».

\section{DISCUSSÃO}

Este estudo apresenta, como principais pontos po- sitivos, o debruçar sobre um tema pouco explorado, particularmente numa população idosa, e ter utilizado uma amostra aleatorizada duma população inscrita nos cuidados de saúde primários.

As suas principais limitações devem-se à não avaliação das comorbilidades dos participantes, à sub-representação na amostra dos indivíduos mais idosos ( $>80$ anos), à aplicação de um questionário de hetero-preenchimento, à diferença de idades significativa entre os entrevistadores e entrevistados (o que pode ter condicionado as respostas obtidas dada a temática sensível que foi explorada), ao número elevado de não-respondentes e ao facto de o número de participantes ter ficado aquém do inicialmente previsto. Adicionalmente teria sido interessante explorar outras características dos participantes, como a sua religião.

A maior importância atribuída à vida sexual pelo género masculino foi acompanhada, neste grupo, pela presença de uma vida sexual mais ativa e de uma maior frequência da atividade sexual. Outros autores verificaram que o género masculino, mesmo em idade geriátrica, se mantém mais sexualmente ativo do que o género feminino (amostra de conveniência constituída por idosos residentes nas suas habitações ou em lar); ${ }^{14}$ e que a maioria dos idosos (amostra de conveniência de idosos residentes em lares) se mantinha sexualmente ativa, com predomínio do género masculino, e apresentavam satisfação na atividade sexual. ${ }^{15}$ Num estudo realizado numa população de idosos nos Estados Unidos, aos 75 anos, 17\% das mulheres e $39 \%$ dos homens tinham uma vida sexual ativa. ${ }^{16}$

Apesar dos homens terem uma vida sexual mais ativa do que as mulheres, não se encontraram diferenças estatisticamente significativas quanto à satisfação com a vida sexual entre os dois géneros.

A diminuição da importância atribuída à vida sexual que se verifica com o avançar da idade pode ter várias causas: alterações fisiológicas naturais, problemas de saúde que frequentemente surgem nos idosos, fatores psicológicos, disfunção sexual feminina ou masculina. ${ }^{17} \mathrm{O}$ envelhecimento é acompanhado de alterações 
hormonais que levam a uma lentificação da resposta sexual e a uma diminuição da intensidade do prazer sexual. Nos homens regista-se uma diminuição da testosterona livre, o que contribui para eventuais problemas de disfunção eréctil. ${ }^{7}$ Há, porém, estudos que sugerem um aumento do interesse nas relações sexuais pelos homens idosos, ${ }^{16}$ provavelmente devido à existência de fármacos eficazes e publicitados para a disfunção eréctil (inibidores da fosfodiesterase-5). Nas mulheres, a diminuição dos níveis de estrogénios após a menopausa condiciona atrofia vaginal com consequente dispareunia e diminuição da intensidade do orgasmo. ${ }^{18}$

Relativamente à associação entre idade e satisfação com a vida sexual não foram identificadas diferenças estatisticamente significativas entre os grupos etários. Teria sido pertinente conhecer alguns dos possíveis fatores confundidores desta associação como as patologias/comorbilidades dos entrevistados e dos companheiros/parceiros sexuais, os antecedentes médico-cirúrgicos e a terapêutica (medicamentosa) em curso.

Os idosos casados/em união de facto atribuíram uma maior importância à vida sexual e mantinham uma vida sexual ativa, apesar de estarem menos satisfeitos com a vida sexual do que os solteiros e viúvos. Fatores expostos previamente podem, em parte, explicar estes resultados. Para as mulheres, $\mathrm{o}$ interesse na vida sexual diminui significativamente quando perdem o seu parceiro/marido. ${ }^{16}$

Quanto aos resultados relativos à qualidade de vida verificou-se uma pontuação mais baixa nas diferentes dimensões do SF-12 na amostra, relativamente à população portuguesa, ${ }^{13}$ devido ao facto das médias desta última não se encontrarem ajustadas para esta faixa etária. A presença de uma vida sexual ativa associou-se de forma estatisticamente significativa a uma maior pontuação nas dimensões «Função Física», «Desempenho Físico» e «Saúde em Geral», em ambos os géneros, e na dimensão «Funcionamento Social» apenas nas mulheres e nas dimensões «Vitalidade» $\mathrm{e}$ «Desempenho Emocional» apenas nos homens.

Em conclusão, a sexualidade é uma componente fundamental da saúde e da qualidade de vida das pessoas, sem restrição de faixa etária. O género masculino atribui uma maior importância à vida sexual e mantém-se sexualmente mais ativo, apesar de não se verifica- rem diferenças significativas entre os géneros quanto à satisfação com a vida sexual. Com o avançar da idade decresce a importância atribuída à vida sexual. O estado civil dos idosos parece influenciar a importância atribuída à vida sexual, bem como a manutenção de uma vida sexualmente ativa e a satisfação daí obtida. Em ambos os géneros, a presença de uma vida sexual ativa associa-se a uma melhor qualidade de vida, traduzida numa melhor pontuação em diferentes dimensões avaliadas.

\section{AGRADECIMENTOS}

A Carla Silva, Iryna Mykolyshyn, Manuel Henriques, Pedro Sousa e Rui Amendoeira por terem participado na realização do protocolo e na recoIha inicial dos dados.

Ao Professor Doutor Pedro Lopes Ferreira por ter autorizado a utilização do questionário SF-12 e por, generosamente, ter realizado o cálculo das dimensões da amostra.

\section{REFERÊNCIAS BIBLIOGRÁFICAS}

1. Ware JE, Snow KK, Kosinski M, Gandek B. SF-36 health survey: manual and interpretation guide. Boston, MA: The Health Institute, New England Medical Center; 1993.

2. Ware JE Jr, Gandek B. Overview of the SF-36 Health Survey and the International Quality of Life Assessment (IQOLA) Project. J Clin Epidemiol. 1998;51(11):903-12.

3. Ferreira PL. Criação da versão portuguesa do MOS SF-36. Parte I: adaptação cultural e linguística [Development of the Portuguese version of MOS SF-36. Part I: cultural and linguistic adaptation]. Acta Med Port. 2000;13(1-2):55-66. Portuguese

4. Ferreira PL. Criação da versão portuguesa do MOS SF-36. Parte II: testes de validação [Development of the Portuguese version of MOS SF36. Part II: validation tests]. Acta Med Port. 2000;13(3):119-27.

5. DeLamater J. Sexual expression in later life: a review and synthesis. J Sex Res. 2012;49(2-3):125-41.

6. Trudel G, Boyer R, Villeneuve V, Anderson A, Pilon G, Bounader J. The marital life and aging well program: effects of a group preventive intervention on the marital and sexual functioning of retired couples. Sexual Relation Ther. 2008;23(1):5-23.

7. Taylor A, Gosney MA. Sexuality in older age: essential considerations for healthcare professionals. Age Ageing. 2011;40(5):538-43.

8. Fernandes L. A sexualidade no idoso. Rev Fac Med Lisboa. 2006;11(4): 225-31.

9. Baldwin K, Ginsberg P, Harkaway RC. Under-reporting of erectile dysfunction among men with unrelated urologic conditions. Int J Impot Res. 2003;15(2):87-9.

10. Ponholzer A, Roehlich M, Racz U, Temml C, Madersbacher S. Female sexual dysfunction in a healthy Austrian cohort: prevalence and risk factors. Eur Urol. 2005;47(3):366-75.

11. Thomas HN, Hess R, Thurston RC. Correlates of sexual activity and satisfaction in midlife and older women. Ann Fam Med. 2015;13(4):33642. 
12. Rohde G, Berg KH, Haugeberg G. Perceived effects of health status on sexual activity in women and men older than 50 years. Health Qual Life Outcomes. 2014;12:43.

13. Ferreira PL, Ferreira LN, Pereira LN. Medidas sumário física e mental de estado de saúde para a população portuguesa [Physical and mental summary measures of health state for the Portuguese population]. Rev Port Saúde Pública. 2012;30(2):163-71. Portuguese

14. Carreira C]. Sexualidade na terceira idade: um estudo comparativo [Dissertation]. Lisboa: Universidade Lusófona de Humanidades e Tecnologias; 2012.

15. Valente R. "Sinto logo existo!..." - Estudo sociológico sobre sexualidade na terceira idade [Internet]. In: VI Congresso Português de Sociologia, Faculdade de Ciências Sociais e Humanas da Universidade Nova de Lisboa, 25 a 28 de junho de 2008. Available from: http://historico.aps.pt/vicongresso/pdfs/72.pdf

16. Lindau ST, Gavrilova N. Sex, health, and years of sexually active life gained due to good health: evidence from two US population based cross sectional surveys of ageing. BMJ. 2010;340:c810.
17. Gott $M$, Hinchliff S. How important is sex in later life? The views of older people. Soc Sci Med. 2003;56(8):1617-28.

18. Latif EZ, Diamond MP. Arriving at the diagnosis of female sexual dysfunction. Fertil Steril. 2013;100(4):898-904.

\section{CONFLITO DE INTERESSES}

Os autores declaram não ter quaisquer conflitos de interesse.

\section{FINANCIAMENTO}

O trabalho apresentado não foi objeto de qualquer tipo de financiamento externo. Todos os encargos foram suportados pelos autores.

\section{ENDEREÇO PARA CORRESPONDÊNCIA \\ Mariana Cambão \\ E-mail: marianacambao@gmail.com \\ http://orcid.org/0000-0001-9404-9478}

Recebido em 23-11-2016

Aceite para publicação em 19-11-2018

\section{ABSTRACT}

\section{QUALISEX: STUDY OF THE ASSOCIATION BETWEEN QUALITY OF LIFE AND SEXUALITY IN THE ELDERLY IN PORTO}

Objectives: To characterize sexuality in elderly people of an urban area of Portugal and explore its relationship with quality of life. Type of study: Observational, cross-sectional and descriptive study, with analytical component.

Local: Family Health Unit Ramalde, West Porto Group of Health Centres.

Population: Patients enrolled in the Family Health Unit Ramalde, aged 65 years or over.

Methods: A random sample was obtained using the electronic tool available at www.random.org. The sample size was calculated using OpenEpi ${ }^{\circledR}$. Users were invited to participate in the study by telephone. For those who agreed to participate, a personal interview was organised using a questionnaire structured in two parts: one prepared by researchers (sociodemographic variables and variables related to sexuality); another corresponding to the SF-12 questionnaire. Data analysis included descriptive and inferential statistics, using the SPSS ${ }^{\circledR}$ v. 24 program.

Results: The sample included 213 users, mostly women (59.2\%), with a mean age of 73 years (standard deviation=5.96). Half of the users had active sex life (mostly men) and $75 \%$ reported being satisfied with their sex life. Males attributed a greater importance to their sex life. There was an association between an active sex life and some dimensions of the SF- 12 in both genders. Women's satisfaction with their sex life was associated to the 'mental health' dimension.

Conclusion: This pioneering study focused on a topic that has been neglected. The importance of sexuality in an age that is sometimes considered 'asexual' has been demonstrated, as well as its impact on the quality of life, thus reinforcing the relevance of addressing these issues in primary care consultations.

Keywords: Aged; Quality of life; Sexuality. 